\title{
Aortic erosion occurring in over 5 years after Amplatzer septal Occluder implantation for secundum atrial septal defect: a case report
}

Yasuko Onakatomi ${ }^{1}$, Toshihide Asou', Yuko Takeda', Hideaki Ueda², Motohiko Goda ${ }^{3{ }^{*}}$ and Munekata Masuda ${ }^{3}$

\begin{abstract}
Background: Aortic erosion is a serious complication that usually occurs shortly after Amplazter Septal Occluder (ASO) implantation for atrial septal defect (ASD).

Case presentation: A seven-year-old girl was diagnosed with secundum ASD without symptoms. Transesophageal echocardiography (TEE) showed a defect of $20 \mathrm{~mm}$ in diameter in the fossa ovalis without aortic rim. An ASO device of $24 \mathrm{~mm}$ in diameter was selected and electively implanted. The "A-shape" of the device was confirmed by intraoperative TEE, a landmark finding indicating the proper implantation of ASO in patients without aortic rim. After an uneventful postoperative course of 5 years and 10 months, she was transferred to our unit due to cardiogenic shock. Her echocardiogram in emergency room showed pericardial effusion with collapsed right ventricle. Given her history of ASO and the observation of the sequentially increasing pericardial effusion, we diagnosed her with acute cardiac tamponade due to aortic erosion. Emergency pericardiotomy was then performed to improve the hemodynamic condition. Fresh clots were found, so we immediately prepared the cardiopulmonary bypass circuit and explored the damage to the aorta, in which the clots had accumulated. Bleeding suddenly started when the clots were removed. We then inserted the cannulae for perfusion and venous drainage. The clots were removed, and tears were found in both the lateral side of the ascending aorta and the right atrial wall. Intraoperative TEE showed that an edge of the ASO device was directly touching the aortic wall and the Doppler color-flow imaging showed blood flow through this lesion. The erosive lacerations of both the ascending aorta and right atrium were detected from the inside after achieving cardioplegic cardiac arrest. The ascending aorta was obliquely incised, and the laceration was closed from inside the aortic root. The postoperative course was uneventful. She has been doing well for 5 years since the surgery.
\end{abstract}

Conclusions: We experienced and successfully treated a rare case of acute cardiac tamponade caused by aortic erosion 5 years and 10 months after ASO implantation.

Keywords: Atrial septal defect, Erosion, Amplazter septal occluder, Cardiac tamponade, Aortic rim

\footnotetext{
* Correspondence: gogomotto@gmail.com

${ }^{3}$ Department of Cardiovascular Surgery, Yokohama City University, Fukuura

3-9, Kanazawa-ku, Yokohama, Kanagawa 236-0008, Japan

Full list of author information is available at the end of the article
}

(C) The Author(s). 2019 Open Access This article is distributed under the terms of the Creative Commons Attribution 4.0 International License (http://creativecommons.org/licenses/by/4.0/), which permits unrestricted use, distribution, and reproduction in any medium, provided you give appropriate credit to the original author(s) and the source, provide a link to the Creative Commons license, and indicate if changes were made. The Creative Commons Public Domain Dedication waiver (http://creativecommons.org/publicdomain/zero/1.0/) applies to the data made available in this article, unless otherwise stated. 


\section{Background}

The Amplazter Septal Occluder (ASO; St. Jude Medical, Plymouth, MN, USA) has been approved for the treatment of secundum atrial septal defect (ASD) since 2005 in Japan. It has been a great boon to a certain number of patients with ASD because it is less invasive and safer than open heart surgery [1]. However, it is associated with a serious complication in aortic erosion, which has been reported to occur in the acute phase, usually within $24 \mathrm{~h}$ after ASO implantation [1].

We herein report a rare case in whom aortic erosion occurred in the late phase after ASO implantation.

\section{Case presentation}

A seven-year-old girl was diagnosed with secundum ASD without symptoms. Transesophageal echocardiography (TEE) showed a defect of $20 \mathrm{~mm}$ in diameter in the fossa ovalis without aortic rim. An ASO device of 24 $\mathrm{mm}$ in diameter was selected and electively implanted. The "A-shape" of the device was confirmed by intraoperative TEE, a landmark finding indicationg the proper implantation of ASO in patients without aortic rim (Fig. 1). After an uneventful postoperative course of 5 years and 10 months, she was transferred to our unit due to cardiogenic shock. Her echocardiogram in emergency room showed pericardial effusion with a collapsed right ventricle. Given her history of ASO and the observation of sequentially increasing pericardial effusion, we diagnosed her with acute cardiac tamponade due to aortic erosion. Emergency pericardiotomy was then performed to improve the hemodynamic condition. Fresh clots were found, so we immediately prepared the cardiopulmonary bypass circuit and then explored the damage of the aorta, in which the clots were accumulated. Bleeding suddenly started when the clots were removed. We then

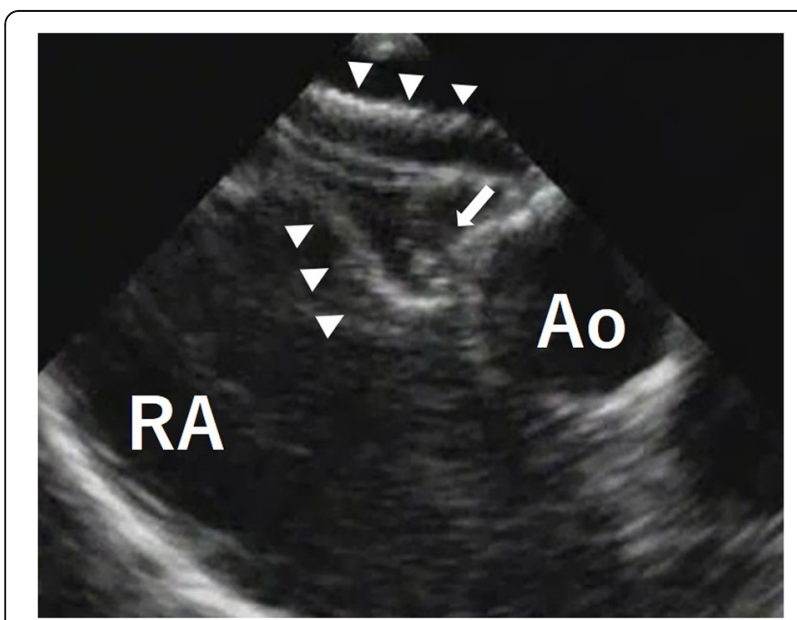

Fig. 1 The transesophageal echocardiogram after ASO implantation revealed the "A-shape" of the device (white arrows) and the absence of an aortic rim

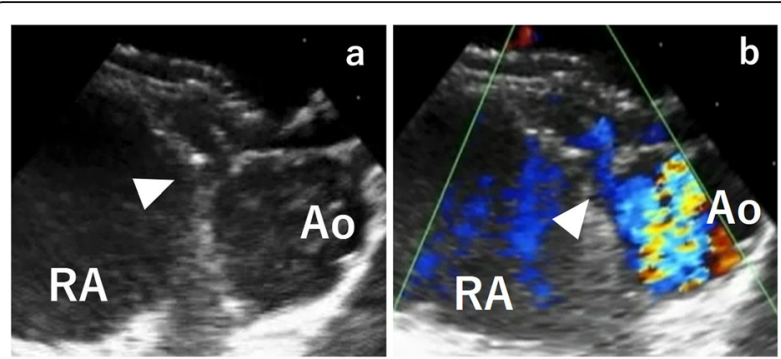

Fig. 2 a, b. The transesophageal echocardiogram during the emergency operation for cardiac tamponade. The edge of the Amplazter septal occluder was touching the aortic wall (a. white arrowhead) and Doppler color-flow imaging showed blood flow through this lesion of the aorta (b. white arrowhead)

inserted the cannulae for perfusion and venous drainage. The clots were removed, and tears were found in both the lateral side of the ascending aorta and right atrial wall. Intraoperative TEE showed that an edge of ASO device was directly touching the aortic wall (Fig. 2a), and Doppler color-flow imaging showed blood flow through this lesion (Fig. 2b). The erosive lacerations of both the ascending aorta (Fig. 3) and right atrium (Fig. 4a) were detected from the inside after achieving cardioplegic cardiac arrest. The ascending aorta was obliquely incised, and both lacerations were closed from the inside of the aortic root and right atrium with 5-0 polypropylene continuous sutures (Fig.4b). We removed the ASO device and performed ASD patch closure. The postoperative course was uneventful. She has been doing well for the 5 years since the surgery.

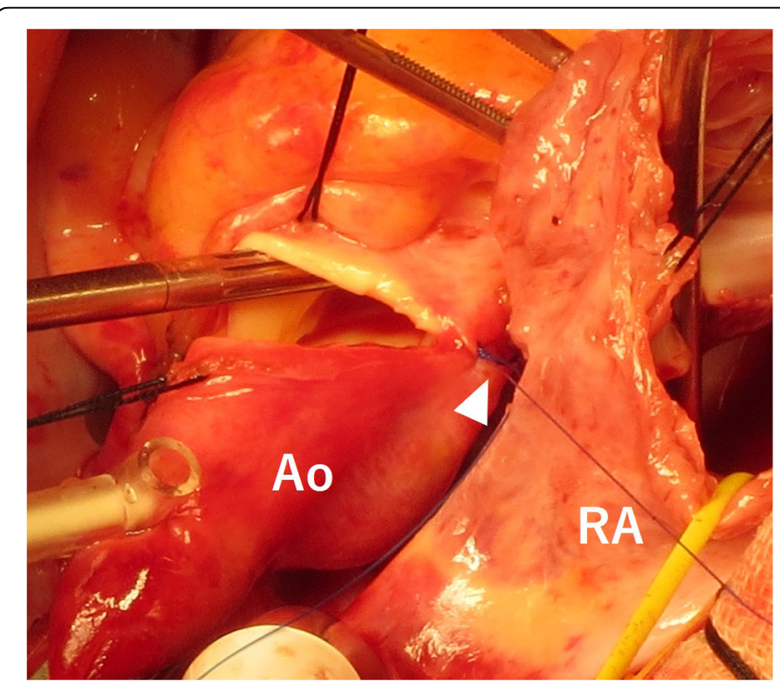

Fig. 3 Operative views. A laceration of the aortic wall facing the right atrium was detected and directly sutured (white arrowhead) 

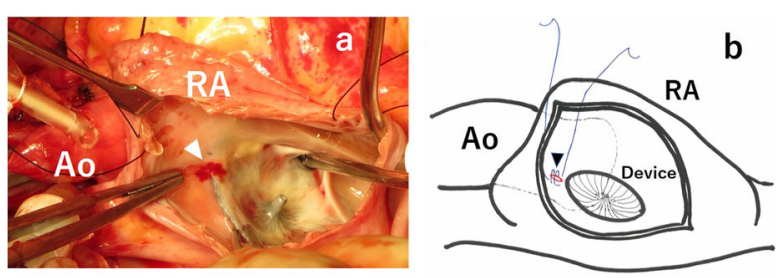

Fig. 4 a, b. Operative views. A laceration induced by Amplazter septal occluder was detected from the inside of the right atrium (a. white arrowhead). The scheme shows that the laceration was closed with 5-0 polypropylene continuous sutures (b. black arrowhead)

\section{Discussion and conclusions}

ASO implantation is less invasive than the surgical procedure with low morbidity and mortality rates and is thus widely indicated in the treatment of the ASD $[1,2]$. However, a major complication with ASO is aortic erosion, which can lead to lethal bleeding. The incidence of this dangerous complication has been reported to be range from 0.1 to $0.3 \%$ [1-3], which is not very frequent. However, the mortality rate was reported to be as high as roughly $20 \%$ [3].

Regarding the mechanism underlying aortic erosion, Amin et al. strongly speculated the absence of the aortic rim of the defect as being involved. They reported that 25 of 28 patients $(89 \%)$ with erosion had an aortic rim of $<5 \mathrm{~mm}$ [4]. A device that oversized and straddled the aortic root was thus believed to carry a risk of causing erosion. When a patient has a deficient aortic rim, the device tends to be placed by straddling it over the aortic root in order to avoid dislodging. Based on this pathogenesis, the fact that the aortic rim of ASD was deficient and the ASD diameter was $20 \mathrm{~mm}$ in our present case suggested a risk of erosion. Given the above, we believe that the ASD in this case should have been surgically treated.

Aortic erosion is most likely to occur 48 to $72 \mathrm{~h}$ after device implantation and rarely in the late phase [3-7]. McElhinney et al. also noted that $1 / 3$ of aortic erosion cases developed it within $24 \mathrm{~h}$ of implantation, and only $6 \%$ developed it over 5 years after implantation [1]. Based on the present and previous findings, we should keep in mind that aortic erosion can occur even years after ASO implantation, and the indication of ASO in cases without a proper aortic rim should be carefully discussed, as ASO implantation is meant to be at least as safe as surgical intervention for ASD.

\section{Authors' contributions}

Concept/design: $Y O$, Data collection: $Y O$, Data analysis/interpretation: $Y O$ Drafting article: YO, MG, Critical revision of article: TA, YT, HU, MM, Approval of article: MM. All authors read and approved the final manuscript.

\section{Funding}

Not applicable.

\section{Availability of data and materials}

The clinical dataset used in this case report are available from the corresponding author on reasonable request.

\section{Competing interests}

The authors declare that they have no competing interests.

Ethics approval and consent to participate

This case report was reviewed and approved by the Institutional Review Board at Kanagawa Children's Medical Center.

\section{Consent for publication}

Informed consent for publication was obtained from the patient's parents.

\section{Author details}

'Department of Cardiovascular Surgery, Kanagawa Children's Medical Center Yokohama, Japan. Department of Cardiology, Kanagawa Children's Medical Center, Yokohama, Japan. ${ }^{3}$ Department of Cardiovascular Surgery, Yokohama City University, Fukuura 3-9, Kanazawa-ku, Yokohama, Kanagawa 236-0008, Japan.

Received: 8 July 2019 Accepted: 2 September 2019

Published online: 06 September 2019

\section{References}

1. McElhinney DB, Quartermain MD, Kenny D, Alboliras E, Amin Z. Relative risk factors for cardiac erosion following transcatheter closure of atrial septal defects a case-control study. Circulation. 2016;133:1738-46.

2. Sadiq M, Kazmi T, Rehman AU, Latif F, Hyder N, et al. Device closure of atrial septal defect: medium-term outcome with special reference to complications. Cardiol Young. 2012;22:71-8.

3. DiBardino DJ, McElhinney DB, Kaza AK, Mayer JE. Analysis of the US Food and Drug Administration manufacturer and user facility device experience database for adverse events involving Amplatzer septal Occluder devices and comparison with the Society of Thoracic Surgery Congenital Cardiac Surgery Databas. J Thorac Cardiovasc Surg. 2009;137:1334-41.

4. Amin Z, Hijazi ZM, Bass JL, Cheatham JP, Hellenbrand WE, et al. Erosion of Amplatzer septal occuluder device after closure of secundum atrial septal defect: review of registry of complications and recommendations to minimize future risk. Cathe Cardiovasc Interv. 2004;63:496-502.

5. Sarris GE, Kirvassilis G, Zavaropoulos P, Belli E, Berggren H, et al. Surgery for complications of trans-catheter closure of atrial septal defects: a multiinstitutional study from the European congenital heart surgeons association. Eur J Cardiothorac Surg. 2010;37:1285-90.

6. Taggart NW, Dearani JA, Hagler DJ. Late erosion of an Amplatzer septal occluder device 6 years after placement. J Thorac Cardiovasc Surg. 2011;142: 221-2.

7. Roberts WT, Parmar J, Rajathurai T. Very late erosion of Amplatzer septal occluder device presenting as pericardial pain and effusion 8 years after placement. Cathe cardiovasc interv. 2013;82:592-84.

\section{Publisher's Note}

Springer Nature remains neutral with regard to jurisdictional claims in published maps and institutional affiliations.

\section{Abbreviations}

